
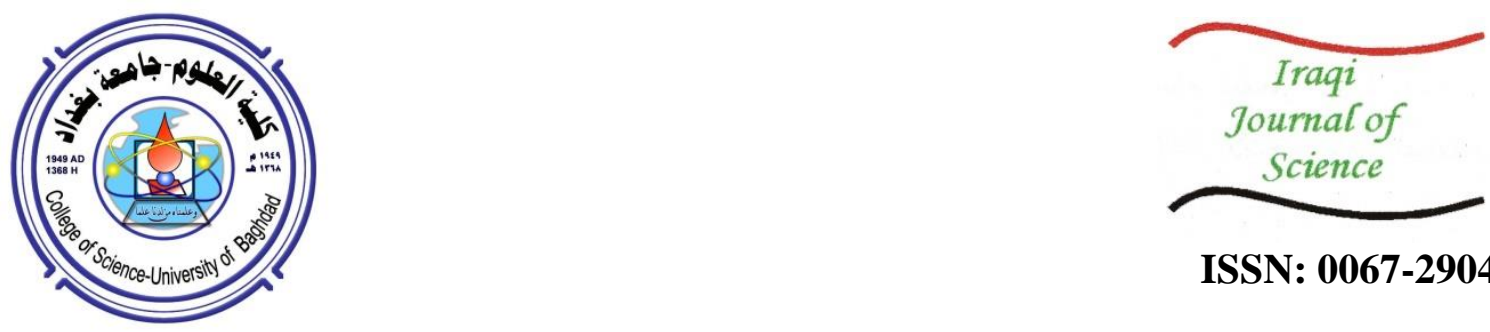

ISSN: 0067-2904

\title{
Dielectric Study of PVC-LiF Composites Films
}

\author{
Ahmad A. Hasan \\ Department of Physics, College of Science, University of Baghdad, Baghdad, Iraq
}

Received: $25 / 1 / 2020$

Accepted: 28/5/2020

\begin{abstract}
PVC-LiF composites films with different lithium fluoride (LiF) concentrations $(0,30,50$, and 70 weight $\%)$ were prepared by using the casting method. This research deals with the investigation of dielectric properties for PVC-LiF composite films as a function of frequency and temperature in the ranges of 100 to $10^{7} \mathrm{~Hz}$ and 293 - $370 \mathrm{~K}$, respectively. The A.C activation energy values estimated from Arrhenius equation were $0.03820,0.3174,0.2009$, and $0.1845 \mathrm{eV}$ for the different PVC-LiF films with different $\mathrm{LiF}$ concentrations, respectively. It was found that the activation energy decreases by increasing $\mathrm{LiF}$ concentration and frequency. The exponent (s) showed a progressive increase with $\mathrm{LiF}$ for PVC-LiF films, while it showed a non-systematic sequence with the increase of temperature. The dependence of the dielectric constant $\left(\varepsilon_{\mathrm{r}}\right)$ and dielectric loss $\left(\varepsilon_{\mathrm{i}}\right)$ on temperature and frequency was investigated for PVC-LiF films with the different LiF concentrations. The dielectric spectrum showed a strong dispersion when $\mathrm{LiF}$ was added to the polymer matrix (PVC) in the whole range of frequency and temperature. The results were interpreted in terms of structural differences caused by the effect of thermal treatment.
\end{abstract}

Keywords: AC Conductivity; dielectric permittivity and relaxation, PVC films.

\section{دراسة عزلية لأغثية متراكبات البولي فينيل كلورايد -ليثيوم فلورايد}

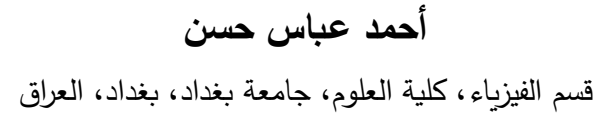

تم تحضير متراكبات البولي فينيل كلورايد-ليثيوم فلورايد PVC-LiF وبتراكيز وزنية مختلفة من الليثيوم فلورايد( 50,30,0 و 70 \% ) بطريقة الصب ـ يعنى هذا البحث باستقصاء الخواص العزلية لأغشية

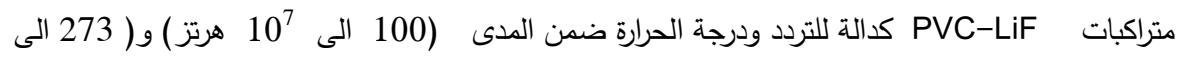

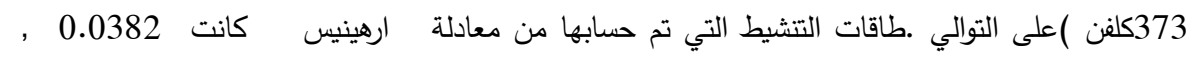
PVC-LiF 0.3174, 0.2009 و 0.1845 الكترون فولت على التوالي لمختلف اغشية المتراكبات التئل ولتراكيز مختلفة من الليثيوم فلورايد. لقد وجد ان طاقة التنتيط تقل مع زيادة تركيز الليثيوم فلورايد والتردد.

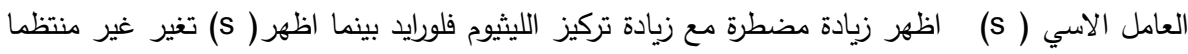

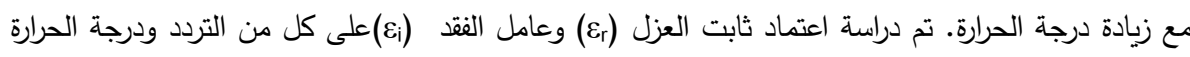

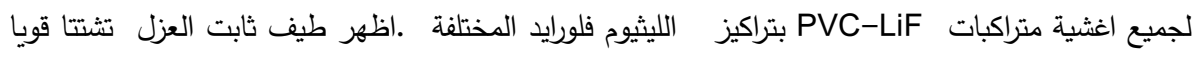

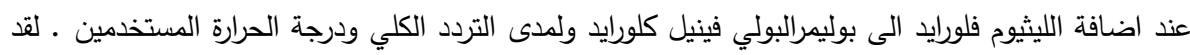
تم تفير النتائج بدلالة تغير التركيب الذي يحدث بتأثير المعاملة الحرارية. 


\section{Introduction}

Polyvinyl chloride (PVC) is the most common polymer with multiple uses which occupies the second place among manufacturing polymers in the world [1]. It is a thermoplastic and linear polymer with an amorphous structure . Plasticization of PVC produces many new properties that are suitable for many applications [2]. Polymerization of vinyl chloride monomer (VCM) produces PVC, The structure of PVC is shown in Figure-1 [3].

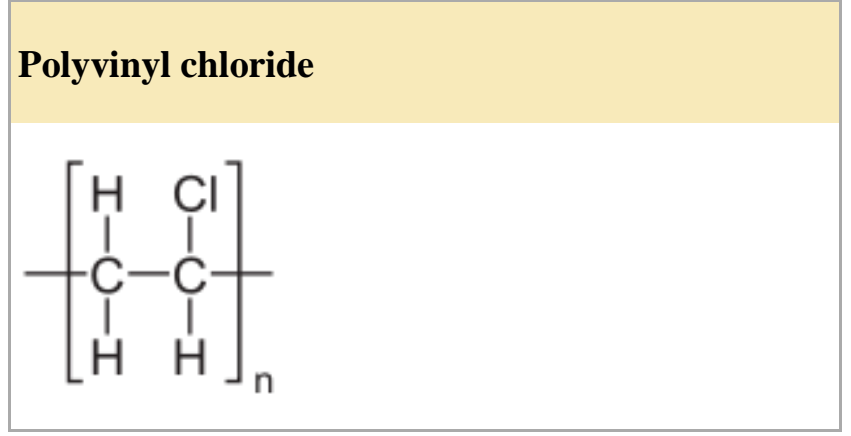

Figure1-The chemical structure of PVC

PVC polymer has a dielectric constant in the range of $3.5-6.5[4,5]$. LiF material is widely used due to the unique optical properties which are appropriate for many applications [6-7]. It is a polycrystalline form of alkali halides family, which has been classified as ionic conductors. The conductivity of $\mathrm{LiF}$ is very law because of the ionic bound resulting from the electrostatic interaction of the oppositely charged ions ( $\mathrm{Li}+, \mathrm{F}-)$ [5]. It can be used in batteries as a layer to improve electron injection [8] .

In these experiments, the electrical conductivity is measured as a function of angular frequency $\omega=2 \pi f$. The full conductivity $\left(\sigma_{t o t}\right)$ at definite frequency and temperature is given by the following equation [9]:

$$
\sigma_{\text {tot }}=\sigma_{a . c}(\omega)+\sigma_{d . c} \quad \mathrm{D}(1)
$$

where the term $\sigma_{d . c}$ is the.D.C conductivity depends strongly on temperature. Many information about the nature of the polarization mechanisms in dielectric and aspects about the interface of the electrodes capacitance as well as the amount electronic conductivity present can be given from the a.c conductivity $\left(\sigma_{\mathrm{ac}}(\omega)\right)$ measurements [10].

The measurements is conducted by measuring the total electrical conductivity $\left(\sigma_{\mathrm{ac}}(\omega)\right)$ as function of angular frequency $\omega=2 \pi f$ where $\mathrm{f}$ is the frequency of an alternating electric field .The total conductivity $\sigma_{\text {tot }}$ is made up from two term as in the following relation : $\sigma_{t o t}=\sigma_{a . c}(\omega)+\sigma_{d . c}$

The first term $\sigma_{d . c}$ is the D.C conductivity which strongly temperature dependence and overbear at low frequencies, however $\sigma_{a . c}$ is the A.C. conductivity which is macerate temperature dependence than $\sigma_{d . c}$ and overbear at high frequency and non temperature dependence then, the empirical relation of frequency dependence on a.c conductivity $\left(\sigma_{a . s}(w)\right)$ shown in many amorphous semiconductors and insulators both inorganic and polymeric material can be written as :

$$
\begin{aligned}
\sigma_{a . c}(\omega) & \propto \omega^{s} \\
\sigma_{a . c} & (\omega)=A \omega^{s}
\end{aligned}
$$

where $\mathrm{A}$ is constant.

The $\sigma_{\mathrm{ac}}$ can be measured by applying the following equation:

$$
\sigma=\frac{t}{R . . A}
$$

where $\mathrm{t}, \mathrm{R}$, and $\mathrm{A}$ are thickness of film, the resistance of sample, and effective area for capacitance, respectively . 
The relation between the AC conductivity and frequency yields three regions . The first is the ionic reign, while the second is the low frequency region which belongs to $\sigma_{\text {d.c }}$ and ascribed to the electrode polarization effects. The third is the high frequency region which belongs to $\sigma_{\text {a.c }}(\omega)$ and ascribed to the relaxation process which is commensurate to $\omega^{\mathrm{S}}$. The exponent is measured from the slope of $\ln \sigma_{\text {a.c }}(\omega)$ versus $\ln (\omega)$ in the high frequency region:

$$
s=\frac{d\left[\operatorname{Ln} \sigma_{a . c}(\omega)\right]}{d[\operatorname{Ln}(\omega)]}
$$

It was found that there are three transport mechanisms of charge carriers that are responsible for the occurrence of conduction, which are [11,12]: (i) transporting by exciting the charge carriers to the extended state near the conduction (C.B) or valence (V) bands, (ii) transporting by exciting the charge carriers into localized states at the extremities of the V.B or C.B, and (iii) hopping of the charge carriers near the Fermi level.

Different theories were previously proposed to explain A.C conduction. These include the quantum mechanical tunneling (QMT) theory where the exponent is temperature independent, the correlated barrier hopping $(\mathrm{CBH})$ theory where the exponent $\mathrm{s}$ is reduced with rising temperature and the A.C conductivity occurs by hopping between defect states [13], and the small polaron theory where the exponent grows by increasing temperature. One of the model which was proposed for the interpretation of these relaxation mechanisms, namely the quantum mechanical tunneling (QMT). In this process, the a.c conductivity is linearly dependent on temperature and the exponent $\mathrm{s}$ is frequencydependent, but independent on temperature [12].

It was pointed out that, for non-conducting materials, the a.c conductivity is temperature-dependent and obeys Arrhenius equation [11].

$$
\sigma_{\mathrm{a} . \mathrm{c}}(\omega)=\sigma_{\mathrm{o}} \exp \left(-\mathrm{E}_{\mathrm{a} . \mathrm{c}} / \mathrm{k}_{\mathrm{B}} \mathrm{T}\right)
$$

It was found that when a dielectric material is subjected to an electric field, the dipoles obey the field. The fraction of the energy supplied from the electric field will be absorbed.

The fraction of energy expended on the molecular friction is expressed as dielectric loss [14]. The behavior of the dielectric loss under an alternating current can be expressed in an equivalent circuit by a combination of connected capacitance (C) with resistance (R) [15]. For the parallel connection, the total impedance $(\mathrm{Z})$ will be given by [16].

$$
1 / Z=1 / R_{p}+j \omega C_{p}
$$

When a dielectric material is subjected to a sinusoidal voltage, the current will be composed from two components, which are the conduction current $\left(I_{c}\right)$ and the displacement current $\left(I_{r}\right)$. The former is in the same phase with voltage while the latter is out of phase in $90^{\circ}$ [17].

$$
\mathrm{I}=\varepsilon^{*} \mathrm{C}_{0} \mathrm{dV} / \mathrm{dt}=\omega \mathrm{C}_{0}\left[\varepsilon_{\mathrm{i}}+\mathrm{j} \varepsilon_{\mathrm{r}}\right] \mathrm{V}
$$

The conduction current $t$ and the displacement current components can be given as :

$$
\begin{aligned}
& I_{\mathrm{c}}=j \omega \varepsilon_{\mathrm{r}} \mathrm{C}_{\mathrm{p}} \mathrm{V} \\
& \mathrm{I}_{\mathrm{r}}=\omega \mathrm{C}_{\mathrm{p}} \varepsilon_{\mathrm{i}} \mathrm{V}-
\end{aligned}
$$

Then the real $\varepsilon_{1}$ and imaginary $\varepsilon_{2-}$ parts of the dielectric constant and the loss tangent $\tan \delta$ are expressed as:

$$
\begin{aligned}
\varepsilon_{\mathrm{r}}=\mathrm{C}_{\mathrm{p}} / \mathrm{C}_{0} \\
\varepsilon_{\mathrm{i}}=1 / \mathrm{R}_{\mathrm{p}} \mathrm{C}_{0} \omega-
\end{aligned}
$$

The alternative conductance $\left(\mathrm{G}_{\mathrm{p}}\right)$ is defined as the reciprocal of the resistance at certain frequency in the equivalent parallel circuit (at the same time), which is given by the relation :

$$
\mathrm{G}_{\mathrm{p}}=\sigma_{\mathrm{a} . \mathrm{c}}(\omega) \mathrm{A} / \mathrm{d}
$$

Then $\varepsilon_{\mathrm{r} \text { and }} \varepsilon_{\mathrm{i}}$ can be written as :

$$
\begin{array}{r}
\varepsilon_{\mathrm{i}}=\mathrm{G}_{\mathrm{p}} / \mathrm{C}_{0} \omega \\
\varepsilon_{\mathrm{i}}=\sigma_{\mathrm{a} . \mathrm{c}} / \varepsilon_{\mathrm{o}} \omega
\end{array}
$$

Cole and Cole [18] postulated a standby plot diagram method to defeat any trouble related with the measurements of the dispersion in the frequency extremities (the high and the low range). In order to determine the real and imaginary parts of dielectric constant, two models were proposed. The plot of the real and imaginary parts of dielectric constants yields arc of circles having their centers 
lying below the absicca axis. The diameter of the circle makes an angle $(\alpha \pi / 2)$ with $\varepsilon_{\mathrm{r}}$, thus the polarizability $(\alpha)$ can be determined .The polarizability is equal to 0 the electric field has one relaxation time, while it lies in the range of $0<(\alpha)<1$ when there is a distribution of relaxation time.

This work sheds the light on the dielectric properties of PVC-LiF composites with various $\mathrm{LiF}$ concentrations, including the dielectric constant $\left(\varepsilon_{\mathrm{r}}\right)$ and dielectric loss $\left(\varepsilon_{\mathrm{i}}\right)$. The relation between them was discussed. An attempt was also made to interpret the results in terms of Maxwell- Wagner model. Cole-Cole diagrams were plotted and used to estimate the values of $\alpha$.

\section{Experimental details}

The materials used in this work include PVC as granules from supplied by SIC Company (Saudi Arabia), and LIF supplied by SLC Chemicals Group (England). The casting method was used to obtain films in the form of disk with a diameter of $\sim 5 \mathrm{~cm}$ and thickness of $0.8 \mathrm{~cm}$. These samples were mounted between a pair of electrodes in the sample holder for dielectric measurements. The parallel capacitance and conductance were measured simultaneously by a programmable LCR meter (HP-4274A and HP-4275A models) with an operating frequency that ranges $100-10^{7} \mathrm{~Hz}$, a temperature range of $298-473 \mathrm{~K}$, and a constant voltage of $0.08 \mathrm{~V}$.

\section{Results and Discussion}

In this part of the study we present and discuss the results of A.C conductivity $\sigma_{\text {ac }}(\omega)$, the dielectric constants $\varepsilon_{\mathrm{r}}$, and $\varepsilon_{\mathrm{i}}$ of PVC/LiF composites with different LIF ratios $(0,30,50$, and $70 \%)$. The effects of the weight fraction of the dispersed phase, temperature $(293-373 \mathrm{~K})$, and frequency $\left(100-10^{7} \mathrm{~Hz}\right)$ on $\sigma_{\mathrm{ac}}(\omega)$ are discussed below.

\section{A.C Conductivity measurements}

Figure-2 shows the relation between $\sigma_{t}(\omega)$ and frequency of PVC-LiF composite films with different $\mathrm{LiF}$ concentrations $(0,30,50$, and $70 \% \mathrm{wt})$. The conductivity $\sigma_{\mathrm{t}}(\omega)$ was shown to increase slightly with increase of angular frequency $(\omega)$ in the D.C conductivity range. Hence, the conductivity $\sigma_{t}(\omega)$ is frequency independent but $\sigma_{t}(\omega)$ increased quickly in the higher frequency range, giving rise to the dominance of A.C conductivity.

It is obvious that the frequency variation trend of the net PVC is exponentially frequency dependent in the full frequency range. The addition of LiF to the host polymeric material generates significant changes in behavior of conductivity with frequency. The conductivity of composite samples is frequency independent in low frequency range (below $4 \times 10^{5} \mathrm{~Hz}$ ) and then it is frequency dependent in the range of $>4 \times 10^{5} \mathrm{~Hz}$.

On the other hand, $\sigma_{t}(\omega)$ for all samples is increased by increasing annealing temperature (Ta) for pure and composites samples. The increase of annealing temperature to $373 \mathrm{~K}$ results in make the conductivity of the composites samples $30 / 70,50 / 50$ and $30 / 70 \mathrm{PVC} / \mathrm{LiF}$ are frequency dependence in full frequency range .

The values of the exponent s, which represents the slope of $\sigma_{t}(\omega)$ versus $(\omega)$, are determined and shown in Table-1. It is obvious that the exponent factor values (s) are less than one in the low temperature range which confirmed the hopping mechanism, while s exceeded the unity in high temperature and high $\mathrm{LiF}$ content. It is clear that (s) increases with the increase of $\mathrm{Ta}$ and then decreases at high temperature . Hence, $\mathrm{CBH}$ and the small polaran (SP) are the most suitable models. While s changes in different sequences with $\mathrm{LiF}$ content, i.e ., it decreases upon the first addition of $\mathrm{LiF}$ and then increases with continuous addition, which makes SP and $\mathrm{CBH}$ are the most suitable models, respectively. Small polaron tunneling is the most convenient model when the exponent(s) grows with the rising of temperature. This occurs when the supplementation of the charge carrier to the covalent solid results in a high degree of local lattice distortion, which forms small polaron. Correlated barrier hopping $(\mathrm{CBH})$ is assumed when there is a potential barrier over which the electrons jump [19].

The activation energy $\left(\mathrm{E}_{\mathrm{ac}}\right)$ values were estimated at selected frequencies $(10,100$, and1000kHz) from plotting $\quad \operatorname{Ln} \sigma_{\text {tot }}(\omega)$ versus $(1 / \mathrm{T})$, as shown in Figure-3 and listed in Table-2. The results demonstrate that all the composites samples show one conduction mechanism and, hence, one activation energy. It is obvious that $\mathrm{E}_{\mathrm{AC}}$ values decreases by increasing frequency and $\mathrm{LiF}$ content. Moreover, $\mathrm{E}_{\mathrm{AC}}$ decreases from 0.03820 to $0.0646 \mathrm{eV}$ and from 0.0382 to $0.01845 \mathrm{eV}$ by increasing the angular frequency $(\omega)$ from $100 \mathrm{kHz}$ to $1000 \mathrm{kHz}$ and when $\mathrm{LiF}$ increases from 0 to $70 \%$, respectively. The reduction of $\mathrm{E}_{\mathrm{AC}}$ values with the increase of angular frequency $(\omega)$ is resulting from the increase 
of vibrating energy. This indicates that the conductivity is frequency dependent in full frequency range for high $\mathrm{LiF}$ content. The reduction of $\mathrm{E}_{\mathrm{AC}}$ can be explained as the action of increasing charge carriers supplied from $\mathrm{LiF}$ which leads to the visual decrease of band gap.
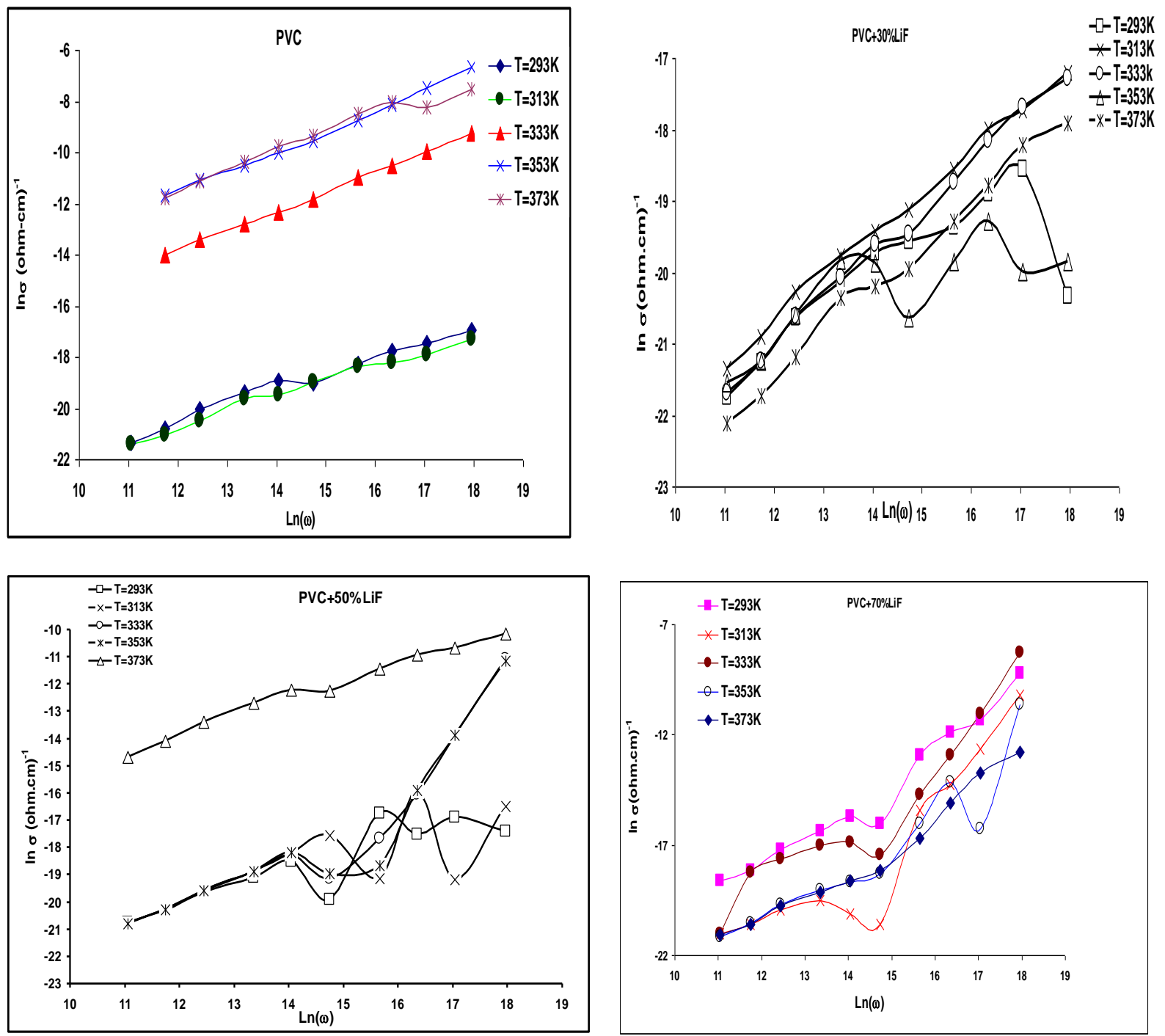

Figure 2-Plot of $\operatorname{Ln} \sigma_{\text {A.C }}$ versus $\ln (\omega)$ of $\mathrm{PVC} / \mathrm{LiF}$ composites with various concentration ratios and different temperatures.

Table 1-The values of the exponent (s) and polrizability $(\alpha)$ of PVC/LiF composites with different concentration ratios and temperatures.

\begin{tabular}{|c|c|c|c|}
\hline \multirow{2}{*}{ LiF ratio \% } & Oven Temperature (K) & $\mathrm{s}$ & $\alpha$ \\
\hline \multirow{4}{*}{0} & 293 & 0.61 & 0.155556 \\
\cline { 2 - 4 } & 313 & 0.59 & 0.155556 \\
\cline { 2 - 4 } & 333 & 0.68 & - \\
\cline { 2 - 4 } & 353 & 0.79 & - \\
\cline { 2 - 4 } & 373 & 0.70 & - \\
\cline { 2 - 4 } & 293 & 0.33 & 0.165556 \\
\cline { 2 - 4 } & 313 & 0.59 & 0.155556 \\
\cline { 2 - 4 } & 333 & 0.63 & 0.133333 \\
\cline { 2 - 4 } & 353 & 0.23 & 0.133333 \\
\hline \multirow{4}{*}{30} & 373 & 0.61 & 0.111111 \\
\hline
\end{tabular}




\begin{tabular}{|c|c|c|c|}
\hline \multirow{4}{*}{50} & 293 & 0.56 & 0.175556 \\
\cline { 2 - 4 } & 313 & 0.51 & 0.165556 \\
\cline { 2 - 4 } & 333 & 1.21 & 0.143333 \\
\cline { 2 - 4 } & 353 & 1.19 & 0.133333 \\
\cline { 2 - 4 } & 373 & 0.61 & 0.121111 \\
\hline \multirow{4}{*}{70} & 293 & 1.34 & 0.145556 \\
\cline { 2 - 4 } & 313 & 1.53 & 0.145556 \\
\cline { 2 - 4 } & 333 & 1.38 & 0.135333 \\
\cline { 2 - 4 } & 353 & 1.29 & 0.133333 \\
\cline { 2 - 4 } & 373 & 1.21 & 0.121111 \\
\hline
\end{tabular}

Table 2-The values of $\mathrm{E}_{\mathrm{ac}}$ for $\mathrm{PVC} / \mathrm{LiF}$ composites with different concentration ratios and frequencies.

\begin{tabular}{|l|l|c|}
\hline LiF content\% & Frequency $(\mathrm{Hz})$ & $\mathrm{E}_{\mathrm{A} . \mathrm{C}}(\mathrm{eV})$ \\
\hline \multirow{3}{*}{0} & 100 & 0.03820 \\
& 1000 & 0.01035 \\
& 10000 & 0.00646 \\
\hline \multirow{3}{*}{30} & 100 & 0.03174 \\
& 1000 & 0.02630 \\
& 10000 & 0.01983 \\
\hline \multirow{3}{*}{50} & 100 & 0.02009 \\
& 1000 & 0.01794 \\
\hline \multirow{3}{*}{70} & 10000 & 0.01345 \\
\hline & 100 & 0.01845 \\
& 1000 & 0.01630 \\
\hline
\end{tabular}

\section{3-2 The Dielectric Constants}

Figure- 4 shows the plot diagram of $\varepsilon_{\mathrm{r}}$ against the angular frequency of PVC-LiF composite films. The spectrum shows that $\varepsilon_{\mathrm{r}}$ changes by rising the annealing temperature and angular frequency . The $\varepsilon_{\mathrm{r}}$ value for the pure PVC decreases with the increase of angular frequency. This is attributed to the domination of the electrode blocking layer, while $\varepsilon_{\mathrm{r}}$ is not influenced by the electronic polarization in high frequency range. It is noticeable that the variation trend of $\varepsilon_{\mathrm{r}}$ with the frequency of the net PVC is different from that of composite samples. It is clear that the domination of the electrode blocking layer becomes more pronounced for all composite samples, i.e., dielectric constant reaches a steady value and not affected by the increase of frequency .

The dielectric constant is increased by rising annealing temperature. Moreover, at frequency $=10^{2} \mathrm{~Hz}$, $\varepsilon_{\mathrm{r}}$ is increased from 911 to 1148 when Ta is increased from 293 to $373 \mathrm{~K}$. However, $\varepsilon_{\mathrm{r}}$ decreases when $\mathrm{Ta}$ increases from 303 to $373 \mathrm{~K}$. The increase of $\varepsilon_{\mathrm{r}}$ value is due to the creation of a high energy barrier . The motion of charge carriers occurred in multiphase materials (PVC-LiF composite films). The trapping and accumulation of charge carriers at available sites, such as defects and interfaces, gives rise to the distortion of the electric field and, hence, to the increase of $\varepsilon_{\mathrm{r}}$. This effect is dominated by the type of the conductivity ( Maxwell-Wagner effect). 

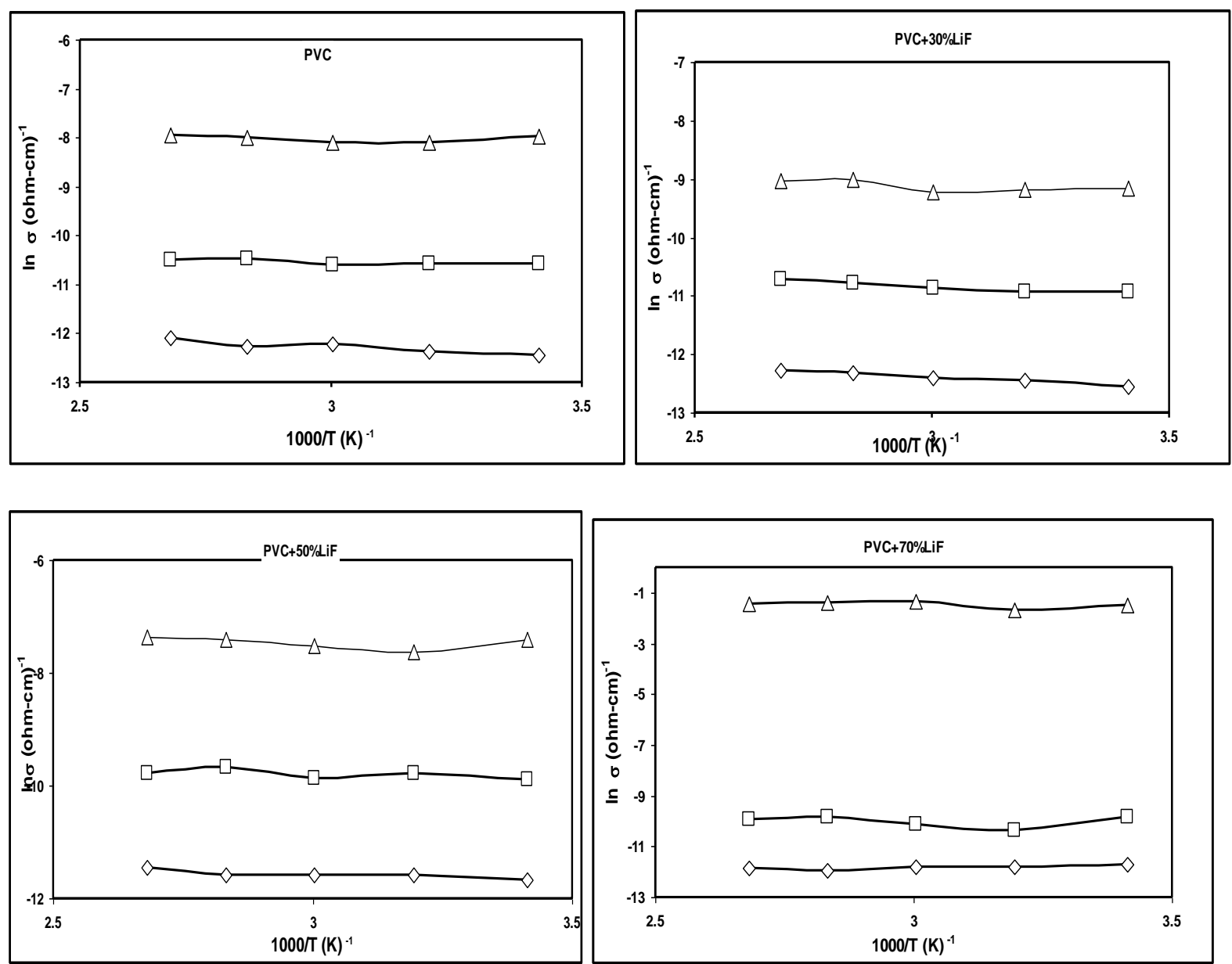

Figure 3- A plot of AC conductivity versus reciprocal temperature (1/T)for PVC-LiF composites.

Figure-5 shows the relation between $\log \varepsilon_{\mathrm{i}}$ and $\ln \omega$. The spectrum shows many peaks which are symmetrical around the angular frequency $\left(\omega_{\mathrm{D}}\right)$ which is corresponded to maximum absorption . Hence, the relaxation time $(\tau)$ can be determined according to the relation $\left(\omega_{D}=1 / \tau\right)$. The results indicated that these peaks are slightly pronounced in the spectrum of the net PVC, but becomes more pronounced by the addition of $\mathrm{LiF}$. The peak of the 70/30 PVC/LiF sample exhibits a shift toward the low frequency side by rising the annealing temperature, while the spectrum of the $50 / 30 \mathrm{PVC} / \mathrm{LiF}$ sample shows more than one peak which vanish by rising the annealing temperature to $373 \mathrm{~K}$. The behavior of the $70 / 30 \mathrm{PVC} / \mathrm{LiF}$ sample is in opposite to that, i.e., there more than one peak appear by rising the annealing temperature. In addition, $\varepsilon_{\mathrm{i}}$ value increases by increasing angular frequency. The explanation of the appearance of more than one peak of $\varepsilon_{i}$ spectrum versus frequency is the formation of more than one phase in the composite samples (i.e. amorphous and crystalline phase). The vanishing of the relaxation peak in the low affirms the dominance of one phase. The foundation of peaks in the dielectric loss spectra postulates relaxing dipoles in all the composite samples. The strength and frequency of relaxation depend on the characteristic property of dipolar relaxation. The addition of $\mathrm{LiF}$ to the net PVC resulted in shifting the relaxation peak toward the lower frequency side. Thus, increasing the relaxation time but further increasing LiF content leads to speeding up the segmental motion by lowering the obtainable free volume. This is proved by the peak shifting toward the higher frequency side, thereby reducing the relaxation time [20]. 


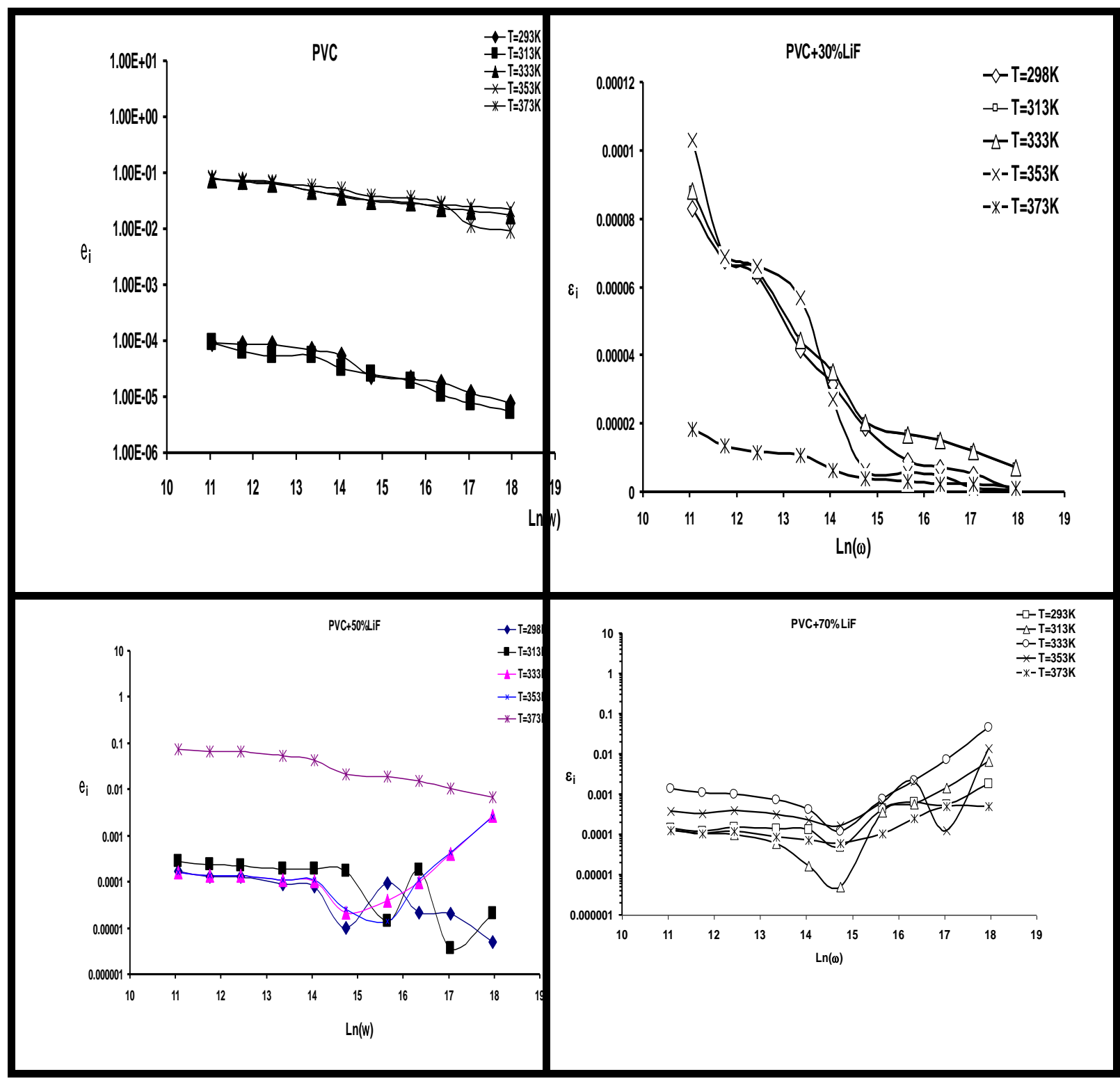

Figure 5-Plot of $\varepsilon_{\mathrm{i}}$ versus $\ln (\omega)$ of $\mathrm{PVC} / \mathrm{LiF}$ composites with different concentration ratios and temperatures.

\subsection{Cole-Cole diagrams}

Cole-Cole diagrams shown in Figure- 6 provide strong evidence of multi-relaxation time in PVC-LiF composite films. The polarizability $(\alpha)$ was measured and the values are listed in Table-1. It is clear that $(\alpha)$ showed a systematic reduction by increasing the annealing temperature, while $(\alpha)$ exhibited a change in an irregular manner with the increase of LiF content in the composite films, i.e., $(\alpha)$ increases by increasing $\mathrm{LiF}$ content to up to $50 \%$ then $(\alpha)$ get to reduce with further increase of $\mathrm{LiF}$ content . This increase in polarizability is related with reaction of the barrier, while the contraction of polarizability is related with the growing intermolecular forces $[21,22]$. 


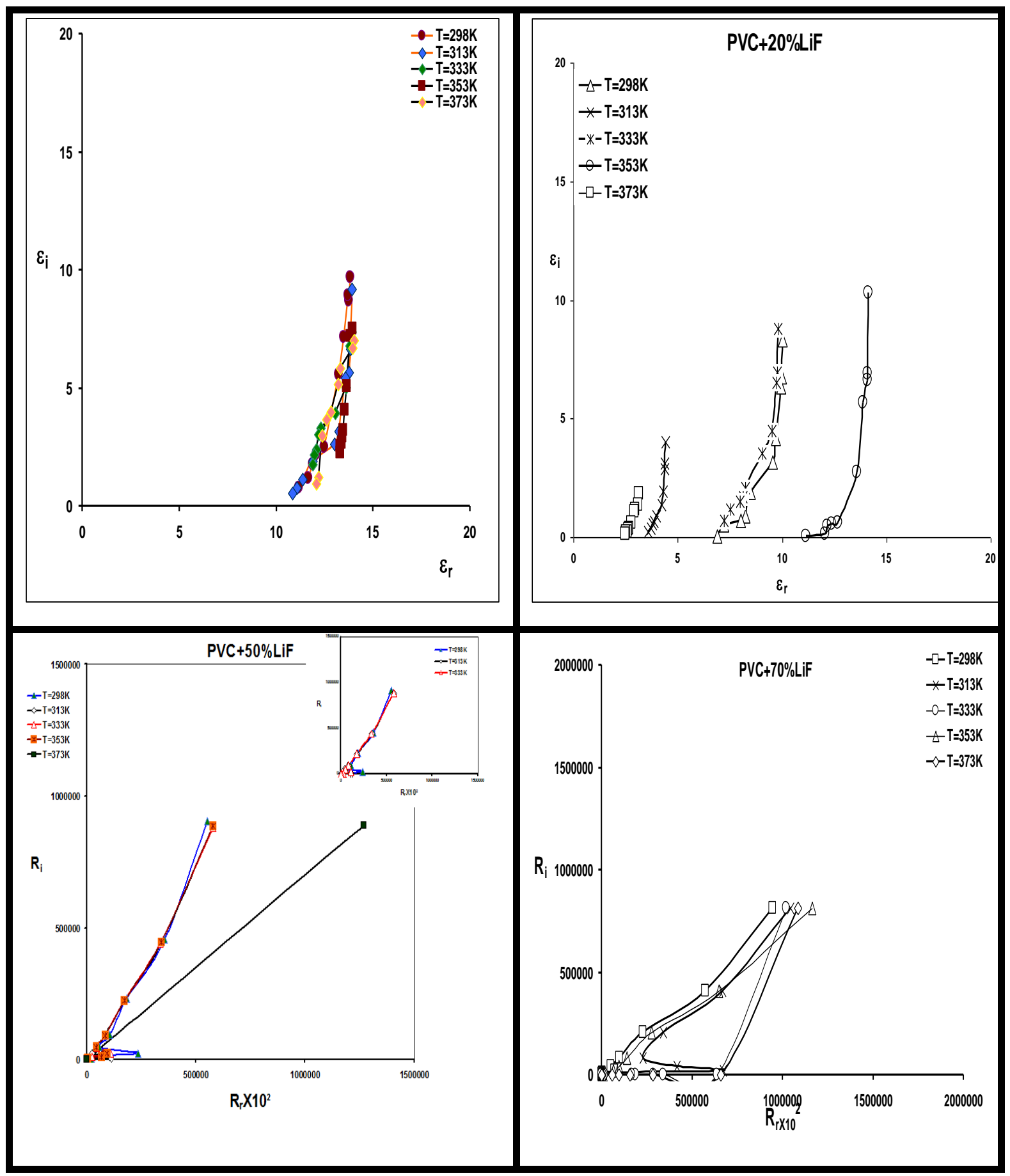

Figure 6-Cole - Cole diagrams of $\mathrm{PVC} / \mathrm{LiF}$ composites with various concentration ratios and temperatures

\section{3-5. Conclusions}

Based on the above mentioned results, one can generally draw the following conclusions:

The addition of LiF to PVC enhances the conductivity. The exponent $\mathrm{s}$ shows a non-systematic variation with the change in $\mathrm{LiF}$ content. The conductivity is frequency dependent in full frequency range by increasing annealing temperature in the range of $313-373 \mathrm{~K}$. The dielectric constant $\varepsilon_{\mathrm{r}}$ increases while $\varepsilon_{\mathrm{i}}$ decreases with the addition of LiF. The dielectric constant $\varepsilon_{\mathrm{r}}$ and $\varepsilon_{\mathrm{i}}$ increase with the increase in annealing temperature and then decrease at high annealing temperature. The low polarizability value $(\alpha<0.5)$ of $\mathrm{PVC} / \mathrm{LiF}$ composites proposes that these composites are more convenient to use as resistors. The reduction of $(\alpha)$ postulates that rising of annealing temperature 
reduces the forces between molecules as a result of the formation of potential barrier, while the increase in $(\alpha)$ with the addition of $\mathrm{LiF}$ postulates an increase in the intermolecular force.

\section{References}

1. L. Claudiu,R. Edina, M. Bogdan, Z. Teodora. 2010. Gheorghe HUPB Sci Bull, Series, 72: 127.

2. M. Subramanian. 2012. " Polymer Testing", Momentum Press.

3. M. Chanda, K. Roy. 2006. " Plastics technology handbook", CRC Press, p.2, London, New York.

4. K.Yoon, Y. Kang, E. Shin and Y. Lee. 2011." Film preparation by melt- or compression-process using poly(vinyl chloride) powder swollen with dimethylformamide", Fibers and Polymers, 12: 29.

5. S. Mhammad. 2001. M.Sc. Thesis, College of Physics, University of Baghdad.

6. P.Heath and P.Sacher. 1986."Quality crystals and optics for laser applications", Applied opt, 15: 937.

7. L. Reale. 2008. "X-ray microscopy of plan cells by using LiF crystal", Microscopy Research and Technique.

8. M. Alvarez. 2008. " Submicron Copper- Low- Density polyethylene conducting composites : Structural, Electrical and percolation Threshold" , Chile.

9. P. Extance, S.R. Elliot and E.A. Davis. 1985. Phys. Rev. B, 32(12): (1985), 8148.

10. A.K. Jonscher, Thin Solid Films, 36: (1976), 1.

11. I.G. Austin and N.F. Mott, 1969. Adv. Polymer, 18(1969): 4.

12. N.F. Mott and E.A. Davis. 1971. Electronic Processes in Non-Crys. Materials, Oxford Univ. Press.

13. S.R .Elliot. 1987. Adv. In. Phys., 36(2): 135.

14. M.A. Omer. 1973. Elementary Solid State Physics, 1st edition, Weslypub. Co.

15. G. Williams, D.C. Watt. 1973. Dielectric Properties of Polymers, Plenum Press. New York.

16. R.A. Fava. 1980. Polymers-Part C: Physical Properties, V 16, Acadimic Press. New York.

17. A.R. Blyth. 1979. Electrical Properties of Polymers, 3rd edition, Cambridge Univ. Press.

18. R.S. Cole and R.H. Cole, J. Chem. 1941. Phys., 9: 34.

19. Von Hipped A R. 1954. Dielectric and Wave (LondonWiley).

20. F. Kremer and A. Schonhals. 2003. (Eds.), Broad Band Dielectric Spectroscopy, Springer-Verlag Berlin Heidelberg, New York

21. Srivastastava K K, Kumar A, Panwar O S and Lakshminarayan K N. 1979. J. Non-Cryst. Solids, 33: 205

22. El Anwar I M, Mohamad A K and Hammad F F. 1999. Egypt.J. Chem. 42: 527-44 